\title{
Efficient Calculation of ROA Tensors with Analytical Gradients and Fragmentation
}

\author{
ANDREAS J. THORVALDSEN, ${ }^{1}$ BIN GAO,${ }^{1}$ KENNETH RUUD, ${ }^{1 *}$ MAXIM FEDOROVSKY, ${ }^{2, \dagger}$ GÉRARD ZUBER,${ }^{2, \$}$ AND WERNER HUG ${ }^{2}$ \\ ${ }^{1}$ Centre for Theoretical and Computational Chemistry, Department of Chemistry, University of Tromsø, Tromsø, Norway \\ ${ }^{2}$ Department of Chemistry, University of Fribourg, Fribourg, Switzerland
}

\begin{abstract}
We present the results of calculations of Raman optical activity spectra of sizable systems from optical tensors of the fragments, the tensors calculated by an analytic approach at the time-dependent Hartree-Fock level of theory. The analytic approach permits large basis sets which, together with the limited geometrical extent of the fragments, obviates the need for the use of London-type orbitals. The implementation of the analytical gradient approach is formulated in the atomic orbital basis by using the elements of the density matrix as variational parameters. This makes the approach directly applicable to linear scaling methods. We do not solve the response equations for the geometrical distortions of the nuclei but determine instead the second-order perturbed densities with respect to the electromagnetic field perturbations. The number of perturbed density matrices that needs to be determined is thus independent of the number of nuclei, making the approach applicable to fragments with many nuclei and with good quality basis sets. Compared to numerical differentiation schemes, the analytical approach is about 10 times faster even for moderately sized molecules.
\end{abstract}

KEY WORDS: raman optical activity; theoretical calculations; analytic methods; basis sets

\section{INTRODUCTION}

Raman optical activity $(\mathrm{ROA})^{1-4}$ is a chiroptical technique that can determine the absolute configuration of chiral molecules also in cases where the chirality is only present in the nuclear wave function due to isotopic substitution., 5 It can, moreover, provide information about the conformation of the molecules and thus the secondary structure of biopolymers in their native environment. ${ }^{7-9}$

As is the case for vibrational circular dichroism (VCD) ${ }^{10-12}$ the absolute configuration of a chiral molecule can either be determined by the comparison of its experimental spectrum with that of a structurally related molecule or by the comparison with the results of an accurate theoretical calculation. ${ }^{6,13,14}$ Until the recent reformulation of the approach for determining vibrational circular dichroism intensities by Coriani et al. ${ }^{15} \mathrm{VCD}$ intensities would, in the traditional formulation of Stephens, ${ }^{16,17}$ only be calculated at a cost comparable with that of a force field calculation. This contrasts with the calculation of the quantities that determines an ROA spectrum, as these quantities have so far required substantial additional computational efforts. This has been because ROA is determined by third-order molecular quantities involving the geometric derivatives of the electric dipole polarizability, the mixed electric dipole-electric quadrupole polarizability, and the mixed electric dipole-magnetic dipole polarizability. Until recently, it was only possible to determine these quantities by numerical geometrical differentiation of the pertinent electronic polarizabilities. ${ }^{18-24}$ This limited the size of systems which could be treated, unless a molecule could be meaningfully subdivided into fragments. ${ }^{25,26}$ Even where such a subdivision was possible, the quality of the basis sets with which computations could be performed was severely limited. Still, molecules of an impressive size are today accessible even to numerical differentiation techniques. ${ }^{27}$

Liégeois, Champagne, and Ruud recently presented the first analytic calculations of the frequency-dependent geometric derivatives of the three polarizabilities that determine ROA.
The scheme was based on an analytic procedure derived for the calculation of the geometric derivatives of the different frequency-dependent polarizabilities ${ }^{28}$ in the framework of time-dependent Hartree-Fock theory in the molecular orbital basis. Significant speedups compared with the numerical differentiation approach were observed, by as much as a factor of 10. A general scheme extended to the density functional level of theory which also includes contributions arising from the use of London atomic orbitals has been described by Thorvaldsen and Ruud. ${ }^{29}$ This scheme has been implemented by Cheeseman and coworkers in the Gaussian program and used in a number of recent applications. ${ }^{30-42}$

The inherent scaling of molecular orbital-based schemes for calculating energies and molecular properties, either at the Hartree-Fock or the density functional level of theory, limits the applicability of a molecular orbital-based computational scheme to systems of small to medium size. To profit from the advances made in linear scaling energy and response theory, ${ }^{43-48}$ an analytic derivative scheme defined in terms of the density matrix in the atomic orbital basis is needed. In the present work, we present the theory for the analytical calculation of the necessary geometric derivatives of the polarizabilities that determine ROA in terms of the density matrix in the atomic orbital basis. The work is based on an open-ended scheme for calculating molecular properties

Contract grant sponsor: This work has received support from the Norwegian Research Council through a Centre of Excellence Grant (Grant No 179568/ V30), a YFF grant to KR (Grant No 162746/V00), as well as through a grant of computer time from the Norwegian Supercomputing Program. Support has also been provided by the Swiss National Science Foundation through research grants to WH (Grants No 200020-103750, 200020-112201, and 200020-120142). ${ }^{\star}$ Correspondence to: Kenneth Ruud, Centre for Theoretical and Computational Chemistry, Department of Chemistry, University of Tromsø, N-9037 Tromsø, Norway. Email: kenneth.ruud@uit.no 
of arbitrary order for a time- and perturbation-dependent atomic basis set. ${ }^{29,49,50}$ The approach is an extension of our recent implementation of analytic frequency-dependent polarizabilities $^{51}$ and hyperpolarizabilities ${ }^{52}$ in terms of the density matrix in the atomic orbital basis, using the linear scaling response solver implementation of Coriani and coworkers for obtaining the necessary perturbed densities. ${ }^{43,53}$

In contrast to Liégeois et al., we choose not to utilize the so-called $2 n+1$ rule. $^{54,55}$ This rule allows the third-order energy corrections that determine ROA tensors to be computed from the first-order perturbed wave functions. For a third-order molecular property, all first-order perturbed wave functions must then be available, ${ }^{55}$ which is not optimal for geometrical perturbations as the number of geometrically perturbed density matrices increases with increasing size of the molecular system. For this reason, we compute the second-order perturbed density matrices arising from the (mixed) electric dipole, electric quadrupole, and magnetic dipole perturbations.

The present implementation of analytical gradients is limited to non-London atomic orbitals. For finite basis sets, the calculated ROA is therefore gauge-origin dependent. ${ }^{20}$ This is a problem for the large molecules for which analytical gradients are advantageous, as most of them represent geometrically extended systems. Such systems can, however, almost always be subdivided into fragments in an obvious way. We have reformulated in the V-tensor formalism, ${ }^{56,57}$ and computationally implemented, ${ }^{58,59}$ an earlier approach $^{25,26}$ for composing ROA tensors of large systems from suitably chosen fragments. The observation of the translational properties of the ROA tensors ${ }^{60,61}$ for individual fragments amounts to distributing the gauge origin over the molecule, and the high quality of the basis sets rendered possible by the use of analytical gradients for small fragments obviates the need to have recourse to London atomic basis functions.

The remainder of the article is organized as follows. In the next section, we first briefly review the formulae for ROA scattering cross sections pertinent to our work. In the third section, we give an account of the theory of our implementation of analytical gradients, with emphasis on the necessary extensions relative to our previous article describing the implementation of polarizability gradients, ${ }^{51}$ together with the details of their calculation. The section on Fragmentation of Molecular Systems presents our Vtensors based approach for combining the ROA tensor gradients of fragments. Computational details are discussed in the fifth section, and the results of example calculations in the Results and Discussion section, where we discuss the computational savings and the relative precision which can be obtained. The Conclusions section contains some concluding remarks and an outlook on the general potential of combining the analytical gradient method with the synthesis of ROA tensors of large systems from fragments.

\section{RAMAN OPTICAL ACTIVITY SCATTERING CROSS SECTIONS}

In contrast to a transmission experiment, light scattering can be measured for different scattering angles $\theta$ and with different polarization schemes. The theoretical measure for Raman scattering into $4 \pi$ is the scattering cross section $\sigma$, and for scattering into an infinitesimal solid angle, the differential scattering cross section $d \sigma(\theta)$. For ROA, either the polarization of the exciting light can be modulated between right and left circular and the intensity of the scattered light measured (incident circular polarization) or the intensity of the two circularly polarized components of the scattered light can be measured for non-circularly polarized exciting light (scattered circular polarization). Outside resonance, the theoretical expressions concur.

Of main practical interest is ROA backward scattering $(\theta=\pi)$. It is determined exclusively by anisotropic invariants of the scattering tensor. Integral scattering can be of interest due to the absence of quadrupole contributions. ${ }^{56}$ For scattered circular polarization backward scattering by an isotropic sample with naturally $(n)$ polarized exciting light, one has for the average value, and the difference of differential scattering cross sections ${ }^{n} d \sigma_{p, L}$ and ${ }^{n} d \sigma_{p, R}$ for vibration $p$, for left $(\mathrm{L})$ and right $(\mathrm{R})$ circularly polarized light, respectively ${ }^{56,62}$

$$
\begin{aligned}
& { }^{n} d \sigma_{p}(\theta)=\frac{1}{2}\left({ }^{n} d \sigma_{p, \mathrm{R}}(\theta)+{ }^{n} d \sigma_{p, \mathrm{~L}}(\theta)\right), \\
& -. \Delta^{n} d \sigma_{p}(\theta)=-\left({ }^{n} d \sigma_{p, \mathrm{~L}}(\theta)-{ }^{n} d \sigma_{p, \mathrm{R}}(\theta)\right), \\
& { }^{n} d \sigma_{p}(\pi)=K_{p}\left(90 a_{p}^{2}+14 \beta_{p}^{2}\right) d \Omega \\
& -\Delta^{n} d \sigma_{p}(\pi)=\frac{4 K_{p}}{c}\left(12 \beta_{G p}^{2}+4 \beta_{A p}^{2}\right) d \Omega
\end{aligned}
$$

where

$K_{p}=\frac{1}{90}\left(\frac{\mu_{0}}{4 \pi}\right)^{2} \omega_{0} \omega_{p}^{3}$

For integral scattering cross sections, one has

$$
\begin{aligned}
& { }^{n} \sigma_{p}=\frac{4 \pi K_{p}}{3}\left(180 a_{p}^{2}+40 \beta_{p}^{2}\right), \\
& -\Delta^{n} \sigma_{p}=\frac{8 \pi K_{p}}{3 c}\left(180 a G_{p}^{\prime}+40 \beta_{G p}^{2}\right) .
\end{aligned}
$$

The minus signs for $\Delta \sigma$ and $\Delta \sigma$ render the standard definition of molecular quantities as left minus right in optical activity compatible with the ROA convention of representing scattering intensities as right minus left. $\omega_{0}$ and $\omega_{p}$ are the pulsations of the exciting and the scattered light, respectively, $c$ is the speed of light, and $\mu_{0}$ is the permeability of vacuum. The formulae are valid for zero Kelvin. For other temperatures, they have to be modified to include appropriate Boltzmann factors. 62

The invariants $I_{p}$ of the scattering tensor, where $I_{p}$ stands for $a_{p}^{2}, \beta_{p}^{2}, a G_{p}, \beta_{G p}^{2}$, or $\beta_{A p}^{2}$, are given in the harmonic approximation for a transition from the initial vibrational state $|i\rangle$ to the final state $|f\rangle$ by

$I_{p}=\left\langle f\left|\boldsymbol{q}_{\mathbf{p}}\right| i\right\rangle^{2} \boldsymbol{L}_{p}^{g} \cdot \boldsymbol{V} \cdot \boldsymbol{L}_{p}^{g}$

$\boldsymbol{q}_{\mathbf{p}}$ is the normal coordinate of vibration $p, \boldsymbol{L}_{p}^{g}$ are the Cartesian-to-normal mode transformation matrix for the nuclear displacements of the $N$ nuclei $a$ in mode $q_{\mathbf{p}}$, and $\boldsymbol{V}$ is a matrix of mono- and dinuclear tensors $\boldsymbol{V}_{\mathbf{a b}}$ : 
$\boldsymbol{L}_{p}^{g}=\left(\begin{array}{c}\boldsymbol{L}_{1, p}^{g} \\ \cdot \\ \dot{\boldsymbol{L}_{a, p}^{g}} \\ \cdot \\ \dot{\boldsymbol{L}_{N, p}^{g}}\end{array}\right), \boldsymbol{V}=\left(\begin{array}{cccccc}\boldsymbol{V}_{11} & \boldsymbol{V}_{12} & \ldots & \boldsymbol{V}_{1 b} & \ldots & \boldsymbol{V}_{1 N} \\ \cdot & \cdot & & \cdot & & \cdot \\ \cdot & \cdot & & \cdot & & \cdot \\ \boldsymbol{V}_{a 1} & \boldsymbol{V}_{a 2} & \ldots & \boldsymbol{V}_{a b} & \ldots & \boldsymbol{V}_{a N} \\ \cdot & \cdot & & \cdot & & \cdot \\ \cdot & \cdot & & \cdot & & \cdot \\ \boldsymbol{V}_{N 1} & \boldsymbol{V}_{N 2} & \ldots & \boldsymbol{V}_{N b} & \ldots & \boldsymbol{V}_{N N}\end{array}\right)$

The elements $\boldsymbol{V}_{a b}$ of $\boldsymbol{V}$ are given by

$\boldsymbol{V}\left(a^{2}\right)_{a b}=\frac{1}{9}\left(\frac{d \alpha_{\mu \mu}}{d \boldsymbol{g}_{a}}\right)_{0}\left(\frac{d \alpha_{v v}}{d \boldsymbol{g}_{b}}\right)_{0}$,

$\boldsymbol{V}\left(a G^{\prime}\right)_{a b}=\frac{1}{9}\left(\frac{d \alpha_{\mu \mu}}{d \boldsymbol{g}_{a}}\right)_{0}\left(\frac{d \boldsymbol{G}_{v v}^{\prime}}{d \boldsymbol{g}_{b}}\right)_{0}$,

$\boldsymbol{V}\left(\beta^{2}\right)_{a b}=\frac{1}{2}\left[3\left(\frac{d \alpha_{\mu v}}{d \boldsymbol{g}_{a}}\right)_{0}\left(\frac{d \alpha_{\mu v}}{d \boldsymbol{g}_{b}}\right)_{0}-\left(\frac{d \alpha_{\mu \mu}}{d \boldsymbol{g}_{a}}\right)_{0}\left(\frac{d \alpha_{\nu v}}{d \boldsymbol{g}_{b}}\right)_{0}\right]$,

$\boldsymbol{V}\left(\beta_{G}^{2}\right)_{a b}=\frac{1}{2}\left[3\left(\frac{d \alpha_{\mu v}}{d \boldsymbol{g}_{a}}\right)_{0}\left(\frac{d G_{\mu v}^{\prime}}{d \boldsymbol{g}_{b}}\right)_{0}-\left(\frac{d \alpha_{\mu \mu}}{d \boldsymbol{g}_{a}}\right)_{0}\left(\frac{d G_{v v}^{\prime}}{d \boldsymbol{g}_{b}}\right)_{0}\right]$,

$\boldsymbol{V}\left(\beta_{A}^{2}\right)_{a b}=\frac{\omega_{0}}{2}\left[\left(\frac{d \alpha_{\mu v}}{d \boldsymbol{g}_{a}}\right)_{0} \varepsilon_{\mu \rho \sigma}\left(\frac{d A_{\rho, \sigma v}}{d \boldsymbol{g}_{b}}\right)_{0}\right]$,

where $\boldsymbol{g}_{a}$ is the Cartesian displacement vector of nucleus $a$. The Einstein convention for summation over repeated Greek indices is used, and the products on the right-hand side are dyads of the Cartesian derivatives of tensor components. The subscript 0 indicates that the derivatives are calculated at zero field strength and at the equilibrium geometry.

The electric dipole-electric dipole, the imaginary part of the electric dipole-magnetic dipole, and the electric dipoleelectric quadrupole polarizabilities $\alpha_{\mu v}, G_{v v}^{\prime}$, and $A_{\rho, \sigma v}$, respectively, are for exact wave functions defined by the sum-over-states expressions

$\alpha_{\mu v}=2 \sum_{j \neq n} \frac{\omega_{j n}}{\omega_{j n}^{2}-\omega_{0}^{2}} \operatorname{Re}\left(\left\langle n\left|\hat{\mu}_{\mu}\right| j\right\rangle\left\langle j\left|\hat{\mu}_{v}\right| n\right\rangle\right)$

$G_{\mu \nu}^{\prime}=-2 \sum_{j \neq n} \frac{\omega}{\omega_{j n}^{2}-\omega_{0}^{2}} \operatorname{Im}\left(\left\langle n\left|\hat{\mu}_{\mu}\right| j\right\rangle\left\langle j\left|\hat{m}_{v}\right| n\right\rangle\right)$,

$A_{\rho, \sigma v}=2 \sum_{j \neq n} \frac{\omega_{j n}}{\omega_{j n}^{2}-\omega_{0}^{2}} \operatorname{Re}\left(\left\langle n\left|\hat{\mu}_{\rho}\right| j\right\rangle\left\langle j\left|\hat{\Theta}_{\sigma v}\right| n\right\rangle\right)$,

where we have introduced the operator $\hat{\mu}_{\mu}$ for a component of the electronic contribution to the electric dipole moment, $\hat{m}_{\mu}$ for the magnetic dipole moment, and $\hat{\Theta}_{\mu \nu}$ for the traceless electric quadrupole moment

$\hat{\mu}_{\mu}=\sum_{i} r_{i, \mu}$

$\hat{m}_{\mu}=\frac{1}{2} \sum_{i} \varepsilon_{\mu \rho \sigma} r_{i, \rho} p_{i, \sigma}$

$\hat{\Theta}_{\mu v}=\frac{1}{2} \sum_{i}\left(3 r_{i, \mu} r_{i, v}-r_{i \mu v}^{2}\right)$.

In these equations, we have used atomic units. $|n\rangle$ denotes the electronic ground state and $|j\rangle$ the excited states, respectively, and $\varepsilon_{\mu \rho \sigma}$ is the antisymmetric unit tensor of Levi-Civita.

\section{ATOMIC ORBITAL-BASED SCHEME FOR ANALYTICAL RAMAN OPTICAL ACTIVITY CALCULATIONS}

To find suitable expressions for the calculation of the geometric derivatives of the frequency-dependent dipole polarizability, the mixed electric dipole-electric quadrupole polarizability and the electric dipole-magnetic dipole polarizability appearing in eqs. 15-17, we take as our starting point the molecular gradient of the quasi-energy defined for an atomic orbital basis, which is time dependent and which depends explicitly on the externally applied perturbation (in this case corresponding to displacements of the nuclei) ${ }^{51}$

$Q^{g}=\frac{d}{d g} Q=\left\{\frac{\partial}{\partial g} \tilde{E}(\mathbf{D})-\operatorname{Tr} \mathbf{S}^{g} \mathbf{W}\right\}_{t}$,

where $\{\ldots\}_{t}$ indicates time averaging and $\operatorname{Tr}$ matrix trace. We have also introduced the density matrix $\mathbf{D}$ in the atomic orbital basis, the generalized self-consistent field energy of the system $\tilde{E}(\mathbf{D})$, defined as

$\tilde{E}(\mathbf{D})=h_{\text {nuc }}+v_{\text {nuc }}+\operatorname{Tr}\left(\mathbf{h}+\mathbf{V}-\frac{i}{2} \mathbf{T}+\frac{1}{2} \mathbf{G}(\mathbf{D})\right) \mathbf{D}$,

where we have defined an antisymmetric time-differentiated overlap matrix $\mathbf{T}$

$T_{\mu v}=\left\langle\chi_{\mu} \mid \dot{\chi}_{v}\right\rangle-\left\langle\dot{\chi}_{\mu} \mid \chi_{v}\right\rangle, \quad \dot{\chi}_{\mu}=\frac{d}{d t} \chi_{\mu}$,

the nuclear repulsion energy $h_{\text {nuc }}$, and the operator describing the interaction between the nuclei and the external field $v_{\text {nuc }}$. $\mathbf{h}$ is the conventional one-electron operator containing the kinetic energy and nuclear attraction contributions

$h_{\mu v}=\left\langle\chi_{\mu}\left|-\frac{1}{2} \nabla^{2}-\sum_{K} \frac{Z_{K}}{\left|R_{K}-r\right|}\right| \chi_{v}\right\rangle$,

$\mathbf{V}$ describes the interaction between the electrons and the external fields, and $\mathbf{G}(\mathbf{D})$ is the two-electron interaction, which in the atomic orbital basis can be written as

$G_{\mu v}(\mathbf{D})=\sum_{\rho \sigma} D_{\sigma \rho}\left(g_{\mu v \rho \sigma}-g_{\mu \sigma \rho v}\right)$

with the two-electron integrals being defined as

$g_{\mu \nu \rho \sigma}=\iint \chi_{\mu}^{*}\left(\mathbf{x}_{1}\right) \chi_{v}\left(\mathbf{x}_{1}\right) \frac{1}{r_{12}} \chi_{\rho}^{*}\left(\mathbf{x}_{2}\right) \chi_{\sigma}\left(\mathbf{x}_{2}\right) d \mathbf{x}_{1} d \mathbf{x}_{2}$.

In the specific case of ROA, the field-molecule interaction operator entering into our Hamiltonian is given by

$$
\begin{aligned}
\hat{V}(t)=- & {\left[f \exp \left(-i \omega_{0} t\right)+f^{*} \exp \left(i \omega_{0} t\right)\right] \cdot \hat{\mu} } \\
& -\left[q \exp \left(-i \omega_{0} t\right)+q^{*} \exp \left(i \omega_{0} t\right)\right] \cdot \hat{\Theta} \\
& -\left[-i b \exp \left(-i \omega_{0} t\right)+i b^{*} \exp \left(i \omega_{0} t\right)\right] \cdot \hat{m} .
\end{aligned}
$$

The first bracketed term is the time-dependent external electric field vector (at the origin), parameterized by the complex-valued perturbation strength vector $f$ (amplitude, phase, and polarization). The second bracket is the electric field gradient (homogeneous), parameterized by $q$ (six components). The last bracket is the magnetic field vector (homogeneous), parameterized by $b$. To avoid imaginary matrix elements, a factor of $(-i)$ has been extracted from $b$. 
In the expression for the quasi-energy gradient eq. 21 , we have also introduced the derivative $\mathbf{S}^{g}$ of the overlap matrix

$S_{\mu v}^{g_{a}}=\frac{\partial S_{\mu v}}{\partial g_{a}}=\frac{\partial}{\partial g_{a}}\left\langle\chi_{\mu} \mid \chi_{v}\right\rangle$

and the energy-frequency-weighted density matrix

$\mathbf{W}=\mathbf{D} \tilde{\mathbf{F}} \mathbf{D}+\frac{i}{2} \dot{\mathbf{D S D}}-\frac{i}{2} \mathbf{D S} \dot{\mathbf{D}}$,

where we have introduced the generalized Fock matrix $\tilde{\mathbf{F}}$ defined as the partial derivative of the energy defined in eq. 22 with respect to the density matrix transposed

$\tilde{\mathbf{F}}=\frac{\partial}{\partial \mathbf{D}^{T}} \tilde{E}(\mathbf{D})=\mathbf{h}+\mathbf{V}-\frac{i}{2} \mathbf{T}+\mathbf{G}(\mathbf{D})$.

As all these matrices are defined in the atomic orbital basis, this provides a very general starting point for frequencydependent response functions, where contributions from both the perturbation dependence and time dependence of the atomic orbital basis are included. We note that the gradient defined in eq. 21 can be considered as a generalization to time-dependent systems and basis sets, of the expression for the conventional geometrical gradient of a molecular system, as introduced by Pulay. ${ }^{63}$

As shown in Ref. ${ }^{51}$, we can obtain higher order, frequencydependent molecular properties by differentiating eq. 21, noting that since the atomic orbitals are independent of the perturbations $f, q$, and $b$ (at least when London orbitals are not used), derivatives of $\mathbf{S}$ involving these perturbations will vanish. Differentiating eq. 21 with respect to the electric field amplitude $f^{*}$ (considering $f$ and $f^{*}$ as independent), we thus get

$Q^{g f^{*}}=\frac{d}{d f^{*}} Q^{g}=\left\{v_{\mathrm{nuc}}^{g f^{*}}+\operatorname{Tr} \mathbf{V}^{g f^{*}} \mathbf{D}+\mathbf{h}^{g} \mathbf{D}^{f^{*}}+\mathbf{G}^{g}(\mathbf{D}) \mathbf{D}^{f^{*}}-\mathbf{S}^{g} \mathbf{W}^{f^{*}}\right\}_{t}$

where we have used that since $g$ are static geometry displacements, the matrix $\mathbf{T}^{g}$ is zero, and that only $v_{\text {nuc }}$ and $\mathbf{V}$ depend on $f^{*}$, and that $\operatorname{Tr} \mathbf{G}^{g}\left(\mathbf{D}^{f^{*}}\right) \mathbf{D}=\operatorname{Tr} \mathbf{G}^{g}(\mathbf{D}) \mathbf{D}^{f^{*}}$. In $Q^{g f^{*}}$, we have omitted the contribution $\operatorname{Tr} \mathbf{V}^{g} \mathbf{D}^{f^{*}}$, since $\mathbf{V}$ is zero in the absence of perturbing fields (i.e., $f=q=b=0$ ). We will however include this contribution when differentiating $Q^{g f^{*}}$ further.

Differentiating the expression for the dipole gradient in eq. 31 a second time, with respect to either of $f$, $q$, or $b$, using the fact that higher order derivatives of the symmetric and antisymmetric overlap matrices involving electric dipole, electric quadrupole, and magnetic dipole perturbations will vanish (since London atomic orbitals are not used in these calculations), as will also all contributions being differentiated three times with respect to the external perturbations, we obtain the final expressions for the geometry derivatives of the three frequency-dependent polarizabilities. These polarizability gradients are then given by the expressions

$$
Q^{g f^{*}}=\left\{\operatorname{Tr}\left(\mathbf{V}^{g f} \mathbf{D}^{f^{*}}+\mathbf{V}^{g f^{*}} \mathbf{D}^{f}+\mathbf{h}^{g} \mathbf{D}^{f^{*}}+\mathbf{G}^{g}\left(\mathbf{D}^{f}\right) \mathbf{D}^{f^{*}}+\mathbf{G}^{g}(\mathbf{D}) \mathbf{D}^{f f^{*}}-\mathbf{S}^{g} \mathbf{W}^{f f^{*}}\right)\right\}_{t},
$$

$Q^{g f^{*}}=\left\{\operatorname{Tr}\left(\mathbf{V}^{g q} \mathbf{D}^{f^{*}}+\mathbf{V}^{g f^{*}} \mathbf{D}^{q}+\mathbf{h}^{g} \mathbf{D}^{q f^{*}}+\mathbf{G}^{g}\left(\mathbf{D}^{q}\right) \mathbf{D}^{f^{*}}+\mathbf{G}^{g}(\mathbf{D}) \mathbf{D}^{q f^{*}}-\mathbf{S}^{g} \mathbf{W}^{q f^{*}}\right)\right\}_{t}$,

$Q^{g f^{*}}=\left\{\operatorname{Tr}\left(\mathbf{V}^{g b} \mathbf{D}^{f^{*}}+\mathbf{V}^{f^{*}} \mathbf{D}^{b}+\mathbf{h}^{g} \mathbf{D}^{b f^{*}}+\mathbf{G}^{g}\left(\mathbf{D}^{b}\right) \mathbf{D}^{f^{*}}+\mathbf{G}^{g}(\mathbf{D}) \mathbf{D}^{b f^{*}}-\mathbf{S}^{g} \mathbf{W}^{b f^{*}}\right)\right\}_{t}$
The first of these equations, eq. 32, has previously been derived in the context of coherent anti-Stokes Raman scattering ${ }^{51}$ and analytic calculations of pure vibrational contributions to nonlinear optical properties, ${ }^{52}$ whereas the two polarizability gradients in eqs. 33 and 34 are new and enter in addition when calculating ROA. The response function gradients in eqs. 10-14 relate to the quasi-energy derivatives as

$\frac{d \alpha_{\mu v}}{d g_{a}}=-Q^{g_{a} f_{v} f_{\mu}^{*}}, \frac{d G_{\mu v}^{\prime e}}{d g_{a}}=Q^{g_{a} b_{v} f_{\mu}^{*}}, \frac{d A_{\rho, \sigma v}^{e}}{d g_{a}}=-Q^{g_{a} q_{\sigma v} f_{\rho}^{*}}$.

We note that the only modifications required in passing from calculating the electric polarizability gradients to the calculation of the mixed electric dipole-magnetic dipole and electric dipole-electric quadrupole polarizabilities are new one-electron interaction operators that have previously been derived and implemented in Ref. $^{28}$, as well as the calculation of additional first- and second-order perturbed density matrices, $\mathbf{D}^{b}, \mathbf{D}^{q}, \mathbf{D}^{q f^{*}}$, and $\mathbf{D}^{b f^{*}}$. Whereas the determination of a Raman spectrum requires the calculation of nine perturbed density matrices (three first order and six second-order perturbed matrices), the calculation of ROA according to eqs. 32-34 requires the solution of 12 first-order perturbed densities (three for the dipole moment operator, three for the magnetic moment, and six for the quadrupole operator using the symmetry of the operator), and 33 second-order equations (six second order in the electric fields, nine mixed electric dipole-magnetic dipole second-order perturbed densities, and 18 mixed electric dipole-electric quadrupole perturbed densities), thus 45 response equations in total.

The first- and second-order perturbed density matrices can be obtained from a set of linear equations that can be solved iteratively. ${ }^{29}$ Both the first- and the second-order equations are of the same form, allowing us to use the same solver for determining both the first- and second-order perturbed densities. These equations have been derived previously ${ }^{29}$ and will not be repeated here. We note, however, that the structure of these equations is such that we can in principle use the linear scaling response solver of Coriani et al. for determining the perturbed densities. $^{43}$

In our previous article on the analytic calculation of the ROA invariants, ${ }^{28}$ we used the $2 n+1$ rule to avoid having to determine the second-order perturbed density matrices. The price we had to pay for this was that we instead had to determine the first-order perturbed density matrices with respect to the nuclear displacements. However, as there are three displacements for each atom in the molecule, the number of response equations increases quickly with increasing size of the molecule, and already for molecules with more than six atoms will there be more response equations to be solved when using the $2 n+1$ rule than the $n+1$ scheme used in eqs. $32-34$. As such, the present implementation should be computationally more favorable for the study of ROA in larger molecules.

Another difference in the approach presented here, relative to that published by Liégeois et al., ${ }^{28}$ is that it is formulated fully in the atomic orbital basis, whereas our previous implementation was derived in the molecular orbital basis. In this manner, the approach presented here can be more directly applied to the study of larger molecular systems, taking advantage of the progress in the developments of linear scaling theory for the calculation of molecular properties. Indeed, the formalism presented here is connected to the linear scaling energy $^{53}$ and response theory code ${ }^{43}$ of Jørgensen, Coriani, 
and coworkers. In this work, we will instead use the conventional molecular orbital-based response solver of the Dalton program, to allow for a more direct comparison of the timings obtained using the analytic and numerical schemes. However, this also means that additional speedups can be expected when full advantage is taken of the speedups achievable with a more efficient integral evaluation scheme ${ }^{64}$ as well as linear scaling technology for larger molecular systems.

\section{FRAGMENTATION OF MOLECULAR SYSTEMS Decomposition of Vibrational Intensities}

The product $\boldsymbol{L}_{p}^{g} \cdot \boldsymbol{V} \cdot \boldsymbol{L}_{p}^{g}$ that occurs in the expressions for scattering cross sections represents a reduced invariant ${ }^{57}$ and can be written as a sum of mono- and dinuclear terms ${ }^{56}$

$\boldsymbol{L}_{p}^{g} \cdot \boldsymbol{V} \cdot \boldsymbol{L}_{p}^{g}=\sum_{a, b} \boldsymbol{L}_{a, p}^{g} \cdot \boldsymbol{V}_{a b} \cdot \boldsymbol{L}_{b, p}^{g}=\sum_{a, b} J_{a b, p}=J_{p}$.

If the system under consideration is divided into the fragments $A, B, C$, etc., then one can choose the numbering of the atoms $a$ in such a way that the tensors $\mathbf{V}_{a b}$ form blocks $\mathbf{V}^{X Y}$

$(\mathbf{V})=\left(\begin{array}{cccccc}\boldsymbol{V}^{A A} & \boldsymbol{V}^{A B} & \cdot & \boldsymbol{V}^{A X} & \boldsymbol{V}^{A Y} & . \\ \boldsymbol{V}^{B A} & \boldsymbol{V}^{B B} & \cdot & \boldsymbol{V}^{B X} & \boldsymbol{V}^{B Y} & \cdot \\ \cdot & \cdot & \cdot & \cdot & \cdot & \cdot \\ \boldsymbol{V}^{X A} & \boldsymbol{V}^{X B} & \cdot & \boldsymbol{V}^{X X} & \boldsymbol{V}^{X Y} & \cdot \\ \boldsymbol{V}^{Y A} & \boldsymbol{V}^{Y B} & \cdot & \boldsymbol{V}^{Y X} & \boldsymbol{V}^{Y Y} & \cdot \\ \cdot & \cdot & \cdot & . & . & .\end{array}\right)$,

where for block $V^{\mathrm{XY}}$ in expanded form, one has

$\left(\mathbf{V}^{X Y}\right)=\left(\begin{array}{ccccc}\boldsymbol{V}_{a a}^{X Y} & \boldsymbol{V}_{a b}^{X Y} & \boldsymbol{V}_{a c}^{X Y} & . & . \\ \boldsymbol{V}_{b a}^{X Y} & \boldsymbol{V}_{b b}^{X Y} & \boldsymbol{V}_{b c}^{X Y} & \cdot & \cdot \\ \boldsymbol{V}_{c a}^{X Y} & \boldsymbol{V}_{c b}^{X Y} & \boldsymbol{V}_{c c}^{X Y} & \cdot & \cdot \\ \cdot & \cdot & \cdot & \cdot & \cdot \\ \cdot & . & . & . & .\end{array}\right)$

with $J_{p}$ being the sum of the invariants $J_{p}^{X Y}$ of individual blocks $J_{p}=\sum_{X, Y} J_{p}^{X Y}$

\section{Molecular V-tensors from Fragments}

Our strategy is to replace the $\mathrm{V}$ tensors of the fragments $A, B$, $C$, etc. of a larger, composite system by those of structurally similar groups and in a case where the larger system represents a cluster of distinct molecules by the independently calculated molecules. In either case, neither the orientation nor the position of the structurally similar entities of the large system will coincide with those of the groups we are using for modeling it. Thus, we have to rotate and translate the groups so that the positions of their atoms match as closely as possible those of the equivalent atoms of the composite system.

Rotation of molecular tensors. The alignment of fragments based on quaternion rotation has been discussed in the context of a comparison of nuclear motions in normal modes. ${ }^{65}$ The quaternions derived from least-square fitting the positions of the nuclei can be converted into the appropriate standard rotation matrices, ${ }^{66}$ a procedure which has been implemented in PyVib2. ${ }^{58}$ If $M_{\mu \rho}$ is the matrix for rotating a group from the orientation in which it was originally computed into that required for aligning it with the system to be modeled, then a second-rank molecular tensor $T_{\rho \sigma}$, such as the electric dipole-electric dipole polarizability tensor, is obtained for the new orientation as

$T_{\mu \nu}=M_{\mu \rho} M_{v \sigma} T_{\rho \sigma}$.

The gradients of the second-rank tensors occurring in the expressions for scattering cross sections transform as third-rank tensors and thus

$\frac{\partial T_{\mu v}}{\partial x_{a \lambda}}=M_{\lambda \tau} M_{\mu \rho} M_{v \sigma} \frac{\partial T_{\rho \sigma}}{\partial x_{a \tau}}$.

We are not concerned with rotating the displacement vectors $\boldsymbol{L}_{g}$ of the nuclei as they are calculated for the modeled system as a whole.

Translation of optical activity tensors. The tensors $\boldsymbol{V}_{a b}^{A B}$ are origin dependent because the optical activity tensor $G_{\mu \nu}{ }^{e}$ and the electric dipole-electric quadrupole tensor $A_{\rho, \sigma v}^{e}$ depend, in the presence of a nonvanishing polarizability tensor $\alpha_{\mu \nu}^{e}$, on the origin chosen for their computation. $\alpha_{\mu \nu}^{e}$ itself is, for a neutral system, origin independent.

We assume a global coordinate system with origin $O$ in which all tensors are originally computed. Habitually, the center of charge or the center of mass of the computed entities is placed into $O$, and we will assume the latter to be the case in our terminology, but any point fixed with respect to the nuclei of a group $A$ will fulfill the same purpose. When the group is moved so that the positions of its nuclei coincide with the equivalent nuclei of fragment $A$ of the system $S$ to be modeled, then the center of gravity of group $A$ is moved to the point $O^{A}$ with position vector $R^{A}$ in the coordinate system $O$. Superposing the nuclei of group $B$ onto those of fragment $B$ similarly moves the center of gravity of $B$ to $O^{B}$ with position vector $\boldsymbol{R}^{B}$. In the common coordinate system $O$, the position vectors of all particles of the groups $A$ and $B$ are thus increased by $\boldsymbol{R}^{A}$ and $\boldsymbol{R}^{B}$, respectively.

The way in which the components of the electronic tensors $G_{\mu \nu}^{\prime A}$ and $A_{\rho, \sigma v}^{A}$ depend on the choice of the origin has been discussed in Refs ${ }^{60,61}$. We note that the signs in the formulae given here refer to moving the groups while keeping the origin fixed, rather than moving the origin with respect to the groups. For the group tensors, we also drop, for the sake of simplifying the notation and as no confusion is possible, the explicit mention of their electronic nature.

For group $A$, one has

$$
\begin{aligned}
\left(G_{\mu \nu}^{\prime A}\right)^{S} & =G_{\mu v}^{\prime A}+\Delta G_{\mu v}^{\prime A}=G_{\mu v}^{\prime A}-\frac{\omega_{0}}{2} \varepsilon_{\nu \rho \sigma} R_{\rho}^{A} \alpha_{\mu \sigma}^{A}, \\
\left(A_{\rho, \sigma v}^{A}\right)^{S} & =A_{\rho, \sigma v}^{A}+\Delta A_{\rho, \sigma v}^{A} \\
& =A_{\rho, \sigma v}^{A}+\frac{3}{2} R_{\sigma}^{A} \alpha_{\rho v}^{A}+\frac{3}{2} R_{v}^{A} \alpha_{\rho \sigma}^{A}-R_{\tau}^{A} \alpha_{\rho \tau}^{A}
\end{aligned}
$$

where the superscript $S$ is used to indicate that group $A$ is superposed onto the equivalent fragment of $S$.

The optical activity tensor $\varepsilon_{\mu \rho \sigma}\left(A_{\rho, \sigma v}^{A}\right)$ in the expression for $\boldsymbol{V}\left(\beta_{A}^{2}\right)_{a b}$, eq. 14 , then becomes

$$
\begin{aligned}
\varepsilon_{\mu \rho \sigma}\left(A_{\rho, \sigma v}^{A}\right)^{S}= & \varepsilon_{\mu \rho \sigma} A_{\rho, \sigma v}^{A}+\frac{3}{2} \varepsilon_{\mu \rho \sigma} R_{\sigma}^{A} \alpha_{\rho v}^{A}+\frac{3}{2} \varepsilon_{\mu \rho \sigma} R_{v}^{A} \alpha_{\rho \sigma}^{A} \\
& -\varepsilon_{\mu \rho \sigma} R_{\tau}^{A} \alpha_{\rho \tau \sigma \nu}^{A} .
\end{aligned}
$$

On the right-hand side, the sum $\varepsilon_{\mu \rho \sigma} R_{v}^{A} \alpha_{\rho \sigma}$ in the second of the three translational terms equates to zero because it 
contains the double contraction of an antisymmetric and a symmetric tensor. The expression therefore simplifies to

$\varepsilon_{\mu \rho \sigma}\left(A_{\rho, \sigma v}^{A}\right)^{S}=\varepsilon_{\mu \rho \sigma} A_{\rho, \sigma v}^{A}+\frac{3}{2} \varepsilon_{\mu \rho \sigma} R_{\sigma}^{A} \alpha_{\rho v}^{A}-\varepsilon_{\mu \rho \sigma} R_{\tau}^{A} \alpha_{\rho \tau}^{A}$.

The term $\varepsilon_{\mu \rho \sigma} R_{\tau}^{A} \alpha_{\rho \tau \sigma v}^{A}$ represents a purely antisymmetric tensor so that its double contraction with the symmetric polarizability tensor $\alpha_{\mu v}$ vanishes. For the products of the electronic tensors of two different groups $A$ and $B$, the changes therefore are upon shifting them

$\alpha_{\mu v}^{A} \Delta G_{\mu v}^{\prime B}=-\frac{\omega_{0}}{2} \varepsilon_{v \rho \sigma} R_{\rho}^{B} \alpha_{\mu \nu}^{A} \alpha_{\mu \sigma}^{B}$

$\alpha_{\mu v}^{A} \varepsilon_{\mu \rho \sigma} \Delta A_{\rho, \sigma v}^{B}=-\frac{3}{2} \varepsilon_{\mu \rho \sigma} R_{\rho}^{B} \alpha_{\mu \nu}^{A} \alpha_{\sigma v}^{B}$.

For $B=A$, the expressions equate to 0 because $\alpha_{\sigma v}^{A}=\alpha_{\mu \nu}^{A} \alpha_{\mu \sigma}^{A}$ is a symmetric tensor, and contracting it twice with $\varepsilon_{v \rho \sigma}$ yields zero. This obviously must be so as otherwise the invariants $\beta_{G}^{2}$ and $\beta_{A}^{2}$, eq. 8 and the computed scattering cross sections of whole molecules, would be origin dependent.

The changes in the derivatives of the electronic tensors occurring in $\boldsymbol{V}^{A B}$ are thus

$\left(\frac{d \alpha_{\mu v}^{A}}{d \boldsymbol{g}_{a}}\right)_{0}\left(\frac{d \Delta G_{\mu v}^{\prime B}}{d \boldsymbol{g}_{b}}\right)_{0}=-\frac{\omega_{0}}{2} \varepsilon_{v \rho \sigma} R_{\rho}^{B}\left(\frac{d \alpha_{\mu v}^{A}}{d \boldsymbol{g}_{a}}\right)_{0}\left(\frac{d \alpha_{\mu \sigma}^{B}}{d \boldsymbol{g}_{b}}\right)_{0}$

$\left(\frac{d \alpha_{\mu v}^{A}}{d \boldsymbol{g}_{a}}\right)_{0} \varepsilon_{\mu \rho \sigma}\left(\frac{d \Delta A_{\rho, \sigma v}^{B}}{d \boldsymbol{g}_{b}}\right)_{0}=-\frac{3}{2} \varepsilon_{\mu \rho \sigma} R_{\rho}^{B}\left(\frac{d \alpha_{\mu v}^{A}}{d \boldsymbol{g}_{a}}\right)_{0}\left(\frac{d \alpha_{\sigma v}^{B}}{d \boldsymbol{g}_{b}}\right)_{0}$.

In these and the following equations, it is assumed that nucleus $a$ is part of group $A$ and nucleus $b$ part of group $B$.

The term $\varepsilon_{\mu \rho \sigma} R_{\tau}^{B} \alpha_{\rho \tau \sigma v}^{B}$, eq. 45 , makes no contribution here either, as its derivatives represent a tensor purely antisymmetric in the indices $\mu$ and $v$. We notice that the expressions represent dyads of vectors and that the sequence of the factors matters.

For the tensors $\left(\boldsymbol{V}_{a b}^{A B}\right)^{S}$ with $A$ and $B$ superimposed upon the equivalent fragments of $S$, one then has

$$
\begin{aligned}
& \left(\boldsymbol{V}\left(a^{2}\right)_{a b}^{A B}\right)^{S}=\boldsymbol{V}\left(a^{2}\right)_{a b}^{A B}, \\
& \left(\boldsymbol{V}\left(\beta^{2}\right)_{a b}^{A B}\right)^{S}=\boldsymbol{V}\left(\beta^{2}\right)_{a b}^{A B}, \\
& \left(\boldsymbol{V}\left(a G^{\prime}\right)_{a b}^{A B}\right)^{S}=\boldsymbol{V}\left(a G^{\prime}\right)_{a b}^{A B}, \\
& \left(\boldsymbol{V}\left(\beta_{G}^{2}\right)_{a b}^{A B}\right)^{S}=\boldsymbol{V}\left(\beta_{G}^{2}\right)_{a b}^{A B}-\frac{3 \omega_{0}}{4} \varepsilon_{v \rho \sigma} R_{\rho}^{B}\left(\frac{d \alpha_{\mu v}^{A}}{d \boldsymbol{g}_{a}}\right)_{0}\left(\frac{d \alpha_{\mu \sigma}^{B}}{d \boldsymbol{g}_{b}}\right)_{0}, \\
& \left(\boldsymbol{V}\left(\beta_{A}^{2}\right)_{a b}^{A B}\right)^{S}=\boldsymbol{V}\left(\beta_{A}^{2}\right)_{a b}^{A B}-\frac{3 \omega_{0}}{4} \varepsilon_{\mu \rho \sigma} R_{\rho}^{B}\left(\frac{d \alpha_{\mu v}^{A}}{d \boldsymbol{g}_{a}}\right)_{0}\left(\frac{d \alpha_{\sigma v}^{B}}{d \boldsymbol{g}_{b}}\right)_{0} .
\end{aligned}
$$

Interchanging the symbols $A$ and $B$, and $a$ and $b$, yields the formulae for $\left(V_{b a}^{B A}\right)^{S}$.
From eqs. 50-54, the elements of the matrices $\left(J_{a b, p}^{A B}\right)^{S}$ for groups $A$ and $B$, shifted by $\boldsymbol{R}^{A}$ and $\boldsymbol{R}^{B}$, respectively, for vibration $p$ of the composite system follow as

$\left(J_{a b, p}^{A B}\right)^{S}=J_{a b, p}^{A B}+\Delta J_{a b, p}^{\mathrm{AB}}$,

where

$\Delta J\left(a^{2}\right)_{a b, p}^{A B}=\Delta J\left(\beta^{2}\right)_{a b, p}^{A B}=\Delta J\left(a G^{\prime}\right)_{a b, p}^{A B}=0$,

and

$$
\begin{aligned}
\Delta J\left(\beta_{G}^{2}\right)_{a b, p}^{A B} & =\Delta J\left(\beta_{A}^{2}\right)_{a b, p}^{A B}=\Delta \boldsymbol{V}\left(\beta_{G}^{2}\right)_{a b}^{A B}:\left(\boldsymbol{L}_{a, p}^{A} \boldsymbol{L}_{b, p}^{B}\right) \\
& =-\frac{3 \omega_{0}}{4} \varepsilon_{v \rho \sigma} R_{\rho}^{B}\left(\frac{d \alpha_{\mu v}^{A}}{d \boldsymbol{g}_{a}}\right)_{0}\left(\frac{d \alpha_{\mu \sigma}^{B}}{d \boldsymbol{g}_{b}}\right)_{0}:\left(\boldsymbol{L}_{a, p}^{A} \boldsymbol{L}_{b, p}^{B}\right)
\end{aligned}
$$

where the notation : is used to indicate a double contraction over the dyads involved.

\section{COMPUTATIONAL DETAILS}

Historically, a molecule's structure, its vibrations, and the optical parameters required for the calculation of vibrational intensities were determined by different approaches. ${ }^{67}$ With modern $a b$ initio methods, these quantities can be calculated at an identical level of theory. While this imparts the hallmark of consistency to the results, it is not the most efficient way to proceed. The rendering of the structure and the force field both depend on the potential energy surface in the vicinity of the nuclear equilibrium position, but the gradients of the electronic tensors do not.

In our approach, ${ }^{68}$ the structure and the force field of a molecule are obtained by a calculation optimized for these two quantities irrespective of the electronic tensors. The results are then used for the calculation of the gradients required for Raman and ROA spectra with methods optimized for this purpose. The Dalton program ${ }^{69}$ permits this approach as it allows an independent calculation of the electronic tensor gradients. In this way, we also can separately identify changes in computed spectra due either to the electronic tensors or the vibrational motion of the nuclei.

For the structures and force fields, the r-pc- 2 basis set ${ }^{59,65}$ with the B97-1 functional ${ }^{70}$ was used. The r-pc-2 set (where $r$ stands for "reduced") is derived from Jensen's pc- 2 set ${ }^{71,72}$ by omitting the polarization functions with the highest orbital angular momentum quantum numbers (the d-functions for $\mathrm{H}$, f-functions for $\mathrm{C}, \mathrm{N}, \mathrm{O}$, and $\mathrm{F}$, and the g-functions for $\mathrm{Si}, \mathrm{P}, \mathrm{S}$, and $\mathrm{Cl}$ ) but by keeping the core's triple-zeta description otherwise intact. While significantly smaller than the full pc- 2 set, the rarified set still leads to structures and force fields of high quality. Where electronic tensors were synthesized from fragments, the force field was calculated for the whole molecule. These calculations were performed with the Gaussian program suite. ${ }^{73}$

The Raman and ROA tensors were calculated with a local version of the Dalton program ${ }^{69}$ at the Time-Dependent Hartree-Fock (TDHF) level of theory for an exciting wavelength of $532 \mathrm{~nm}$. Spectra were drawn in PyVib2 ${ }^{58}$ with Lorentzian curves of $3 \mathrm{~cm}^{-1}$ full-width at half maximum height for isotropic and $10 \mathrm{~cm}^{-1}$ for anisotropic contributions, convoluted with a Gaussian instrument band shape of $7 \mathrm{~cm}^{-1}$ full-width at half maximum, as discussed in Ref. ${ }^{5}$ 
As basis sets for the electronic tensor calculations, we used the augmented correlation-consistent polarized valence double-zeta aug-cc-pVDZ set, ${ }^{74,75}$ the DPS set ${ }^{76}$ that is based on the $3-21 \mathrm{G}$ basis set ${ }^{77}$ augmented by diffuse (D) polarization (P) functions and a diffuse shell (S), and the rDPS set ${ }^{78}$ where augmentation is applied to the hydrogen atoms only. The detailed composition of the DPS set is listed in Table 1. We notice that rDPS and DPS are not generally suitable for the calculation of the structure of molecules because they are based on semi-diffuse rather than the standard, more compact polarization functions, and neither does the TDHF approach yield force fields of a quality sufficient for ROA calculations.

Conventional non-London atomic orbitals were used, except for the data required to compare our results with gauge origin-independent London atomic orbital calculations. While these latter calculations were done with numerical gradients, this does not imply that our formalism for analytical gradients is not applicable to London-type atomic orbitals. Rather, the required one- and two-electron integrals are not, at present, implemented in Dalton. In all calculations, the gauge origin was always chosen to coincide with the center of mass of the calculated entity.

The synthesis of the electronic tensors was accomplished with a local version of PyVib2, ${ }^{58}$ expanded to permit the orientation and translation of the tensors of fragments, and their combination into molecular tensors.

All calculations were performed in parallel using 48 processors with the parallelization scheme described in Ref. ${ }^{79}$. They were performed at the local supercomputer installed at the University of Tromsø, which consists of 704 dual CPU $2.66 \mathrm{GHz}$ Intel Xeon X5355 Quad Core processors. To provide comparable timings between the numerical and analytical schemes, performance enhancing features, such as in-core storage of two-electron integrals, ${ }^{80}$ were not used.

\section{RESULTS AND DISCUSSION}

We investigate in this section the difference in the computational times required for numerical and analytical gradients on the one hand, and the quality of the ROA and Raman data and the gain in time that can be obtained by using the small rDPS and DPS basis sets specifically developed for the efficient calculation of electronic tensor gradients ${ }^{76,78}$ on the other, with structures and force fields calculated in the latter case with r-pc-2. Also considered is the feasibility of further speeding up calculations by synthesizing these gradients from tensors computed for fragments as parts of smaller molecules.

The model compounds were the five chiral secondary alcohols shown together with trimethylsilanol in Figure 1, and their trimethylsilylethers. The use of trimethylsilylethers reduces the conformational flexibility of a nonrigid molecule, and these compounds thus serve as a convenient set of reference molecules for which a detailed comparison against experimental observations more easily can be made. As the reference basis set for the electronic tensors, we choose augcc-pVDZ, as it has been shown by Reiher, Liégeois, and Ruud that this basis set gives very good results compared with much larger basis sets. ${ }^{81}$ As previously mentioned, analytical gradients are, at present, not available in Dalton with London orbitals. Results for London orbitals and aug-cc-pVDZ obtained with numerical gradients are therefore included for the secondary alcohols only, but not for their trimethylsilylethers for which calculations would have required too much time. For the sake of consistency, we have consequently used the results for
TABLE 1. Exponents of the DPS basis set for $\mathrm{H}, \mathrm{C}, \mathrm{N}, \mathrm{O}$, and $\mathrm{F}$

\begin{tabular}{|c|c|c|c|}
\hline Shell & \# CGTO & $\alpha_{i}$ & $c_{i}$ \\
\hline \multirow{5}{*}{$\mathrm{s}$} & \multirow{3}{*}{1} & \multicolumn{2}{|l|}{ Hydrogen } \\
\hline & & 5.447178 & 0.156285 \\
\hline & & 0.824547 & 0.904691 \\
\hline & 2 & 0.183192 & 1.000000 \\
\hline & 3 & 0.036000 & 1.000000 \\
\hline $\mathrm{p}$ & 1 & 0.183192 & 1.000000 \\
\hline \multirow{8}{*}{$\mathrm{s}$} & \multirow{4}{*}{1} & Carbon & \\
\hline & & 172.256000 & 0.061767 \\
\hline & & 25.910900 & 0.358794 \\
\hline & & 5.533350 & 0.700713 \\
\hline & \multirow[t]{2}{*}{2} & 3.664980 & -0.395897 \\
\hline & & 0.770545 & 1.215840 \\
\hline & 3 & 0.195857 & 1.000000 \\
\hline & 4 & 0.043800 & 1.000000 \\
\hline \multirow[t]{4}{*}{$\mathrm{p}$} & \multirow[t]{2}{*}{1} & 3.664980 & 0.236460 \\
\hline & & 0.770545 & 0.860619 \\
\hline & 2 & 0.195857 & 1.000000 \\
\hline & 3 & 0.043800 & 1.000000 \\
\hline d & 1 & 0.195857 & 1.000000 \\
\hline \multirow{8}{*}{$\mathrm{s}$} & \multirow{4}{*}{1} & Nitrogen & \\
\hline & & 242.766000 & 0.059866 \\
\hline & & 36.485100 & 0.352955 \\
\hline & & 7.814490 & 0.706513 \\
\hline & \multirow[t]{2}{*}{2} & 5.425220 & -0.413301 \\
\hline & & 1.149150 & 1.224420 \\
\hline & 3 & 0.283205 & 1.000000 \\
\hline & 4 & 0.063900 & 1.000000 \\
\hline \multirow[t]{4}{*}{$\mathrm{p}$} & \multirow[t]{2}{*}{1} & 5.425220 & 0.237972 \\
\hline & & 1.149150 & 0.858953 \\
\hline & 2 & 0.283205 & 1.000000 \\
\hline & 3 & 0.063900 & 1.000000 \\
\hline \multirow[t]{2}{*}{ d } & \multirow[t]{2}{*}{1} & 0.283205 & 1.000000 \\
\hline & & Oxygen & \\
\hline \multirow[t]{7}{*}{$\mathrm{s}$} & \multirow[t]{3}{*}{1} & 322.037000 & 0.059239 \\
\hline & & 48.430800 & 0.351500 \\
\hline & & 10.420600 & 0.707658 \\
\hline & \multirow[t]{2}{*}{2} & 7.402940 & -0.404453 \\
\hline & & 1.576200 & 1.221560 \\
\hline & 3 & 0.373684 & 1.000000 \\
\hline & 4 & 0.084500 & 1.000000 \\
\hline $\mathrm{p}$ & 1 & 7.402940 & 0.244586 \\
\hline & & 1.576200 & 0.853955 \\
\hline & 2 & 0.373684 & 1.000000 \\
\hline & 3 & 0.084500 & 1.000000 \\
\hline d & 1 & 0.373684 & 1.000000 \\
\hline & & Fluorine & \\
\hline $\mathrm{s}$ & 1 & 413.801000 & 0.058548 \\
\hline & & 62.244600 & 0.349308 \\
\hline & & 13.434000 & 0.709632 \\
\hline & 2 & 9.777590 & -0.407327 \\
\hline & & 2.086170 & 1.223140 \\
\hline & 3 & 0.482383 & 1.000000 \\
\hline & 4 & 0.107600 & 1.000000 \\
\hline $\mathrm{p}$ & 1 & 9.777590 & 0.246680 \\
\hline & & 2.086170 & 0.852321 \\
\hline & 2 & 0.482383 & 1.000000 \\
\hline & 3 & 0.107600 & 1.000000 \\
\hline d & 1 & 0.482383 & 1.000000 \\
\hline
\end{tabular}

non-London orbitals as the reference for both the trimethylsilylethers and the secondary alcohols. The comparison of results for the two types of orbitals for the alcohols suggests that 
<smiles>CC(C)COC(=O)[C@H](C)O</smiles>

Isobutyl (S)-(-)-lactate

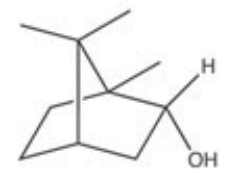

(S)-(-)-Borneol<smiles>CCC[C@@H](O)c1ccccc1</smiles>

(R)-(+)-1-Phenyl-1-butanol<smiles>CC(C)[C@H]1CC[C@@H](C)C[C@H]1O</smiles>

(1R,2S,5R)-(-)-Menthol<smiles>CCCCCC[C@H](C)O</smiles>

(R)-(-)-2-Octanol

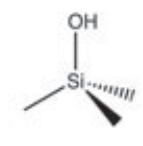

Trimethylsilanol

Fig. 1. Structure of the chiral secondary alcohols and of trimethylsilanol.

non-London orbitals are not a severe limitation for the molecules we consider if the aug-cc-pVDZ basis set is used.

The theoretical error measure we suggest is meaningful for comparing computed data, and we discuss to which extent it reflects the subjective quality of the computed spectra. The conformational equilibria of the model molecules computed with r-pc-2, and their influence on the computed spectra, are rather complex (Hasanova E., Hug W., Lovchik M., unpublished results) and cannot be addressed here. Thus, for the present comparison of theoretical data, the main conformer only was chosen, and a comparison with an experimental spectrum is made only in the case of the trimethylsilylether of menthol, where a single conformer dominates.

\section{Computational Times}

Tables 2 and 3 give, respectively, the relative wall-clock times and the speedup achievable with analytical as compared with numerical gradients, for TDHF calculations of the electronic tensors with the rDPS, DPS, and aug-cc-pVDZ basis set for the five chiral secondary alcohols, and for the rDPS and aug-cc-pVDZ basis sets also for the trimethylsilylethers of the chiral alcohols, and the achiral trimethylsilanol.

TABLE 2. Relative wall-clock times for TDHF calculations with analytical gradients. The unit is the wall-clock time of $227 \mathrm{~s}$ for trimethylsilanol (TMS). For the alcohols, the number of atoms is indicated in brackets. For their trimethylsilylethers, one has to add those of TMS less 3

\begin{tabular}{lccccc}
\hline & & & \multicolumn{3}{c}{ TMS } \\
& & Alcohols & & ethers & \\
& rDPS & DPS & $\begin{array}{c}\text { aug-cc- } \\
\text { pVDZ }\end{array}$ & rDPS & $\begin{array}{c}\text { aug-cc- } \\
\text { pVDZ }\end{array}$ \\
\hline $\begin{array}{l}\text { Trimethylsilanol } \\
\text { (15) }\end{array}$ & 1.0 & & 4.4 & & \\
$\begin{array}{l}\text { Isobutyl lactate } \\
\text { (24) }\end{array}$ & 4.6 & 7.3 & 26.2 & 14.5 & 180.7 \\
$\begin{array}{l}\text { 1-Phenyl-1- } \\
\text { butanol (25) }\end{array}$ & 5.6 & 9.2 & 33.1 & 16.9 & 235.8 \\
$\begin{array}{l}\text { 2-Octanol (27) } \\
\begin{array}{l}\text { Borneol (29) } \\
\text { Menthol (31) }\end{array}\end{array}$ & 3.9 & 7.9 & 27.6 & 17.5 & 184.7 \\
\hline
\end{tabular}

TABLE 3. Reduction of the computational times achieved by using analytical instead of numerical gradients for the alcohols and their trimetylsilylethers. Numerical gradients are simultaneously calculated for London and non-London basis functions

\begin{tabular}{lrccc}
\hline & \multicolumn{3}{c}{ Alcohols } & TMS ethers \\
\hline & rDPS & DPS & $\begin{array}{c}\text { aug-cc- } \\
\text { pVDZ }\end{array}$ & rDPS \\
\hline Isobutyl lactate & 5.7 & 9.5 & 5.5 & 12.1 \\
1-Phenyl-1-butanol & 6.2 & 7.1 & 6.0 & 9.7 \\
2-Octanol & 8.7 & 7.5 & 6.1 & 8.8 \\
Borneol & 8.1 & 10.5 & 6.7 & 9.4 \\
Menthol & 12.9 & 5.5 & 6.8 & 9.3 \\
\hline
\end{tabular}

The tensors of this latter molecule were used for synthesizing those of the ethers. Values for the exponents of the DPS basis set have not yet been determined for silicon, so that no DPS data for the silicon-containing compounds are listed. The wall-clock times for the calculation with numerical gradients and the aug-cc-pVDZ basis set are likewise not available for the trimethylsilylethers due to the excessive computational requirements. Based on the comparison of the available numbers for other basis sets, we assume that they would be an order of magnitude larger than for the alcohols.

Trimethylsilanol with the rDPS basis set, with gradients calculated analytically, yields the shortest computational time, and all other values are given relative to this value. The relative times for the electronic tensors of the trimethylsilylethers, when synthesized from the secondary alcohols and trimethylsilanol, are taken as the sum of the times required for the separate calculations of the smaller molecules. For menthol, the largest secondary alcohol, this yields a relative value of about 64 in the case of analytical gradients and the aug-ccpVDZ basis set. This is less than one-fifth of the value of 330 for the direct calculation of the whole molecule with analytical gradients and of the order of one-fiftieth of the estimated value for the same calculation with numerical gradients.

Twice the speedup of five achieved by synthesizing the tensors using the aug-cc-pVDZ basis set can instead be achieved by calculating the whole molecule with the rDPS basis set and analytical gradients. If, in addition, the tensors 
are synthesized with the rDPS rather than aug-cc-pVDZ basis set, the speedup increases to almost 40 times. Though there are variations in the speedups for the ethers of the different alcohols, these figures reflect the general trend.

The DPS basis set, while slower than the rDPS set, is of interest because it yields a quality of the electronic tensor gradients not far below that achievable with aug-cc-pVDZ. For menthol, DPS is 2.7 times faster than aug-cc-pVDZ with analytical gradients. Similar ratios are expected for the trimethylsilylether of menthol once optimal values for the exponents of the atoms $\mathrm{Si}, \mathrm{P}, \mathrm{S}$, and $\mathrm{Cl}$ become available.

The speedup achieved by the use of analytical gradients, as compared with the numerical ones, depends on the basis set and molecule. From Table 3, one finds an average value of 8.3 for the rDPS set, 8.0 for DPS, but only 6.2 for aug-cc-pVDZ. For the trimethylsilylethers, the average value of the speedup reaches 9.9 for rDPS. While there are substantial variations for individual molecules that cannot readily be attributed either to their size or structure, there appears to be, on average, an increase of the speedup with the size of the molecules, though less so than what one would expect based on the reduction of individual self-consistent field calculations required for numerical gradients.

We notice that a further speedup can be achieved in the analytical computation of gradients by omitting the quadrupole part $\beta_{A}^{2}$ as was done for numerical gradients. ${ }^{82,83} \beta_{A}^{2}$ does not contribute to integral scattering cross sections, ${ }^{56}$ and it is for many bands of little importance also in backscattering spectra. This reduces the number of perturbed densities from 45 to 18 , making the calculation of ROA scattering cross sections only marginally more expensive than that of Raman scattering cross sections.

\section{Quality of Electronic Tensor Gradients}

As seen from eq. 8, the results of an ROA calculation depend simultaneously on the electronic tensor gradients and on the force field. To judge the quality of the electronic tensor gradients obtained with a trial method, the force field is thus kept constant while the approach for the electronic tensors is varied. Differences in the computed spectra can then be attributed to changes in the tensor gradients.

A measure for comparing experimental and theoretical spectra is their similarity. ${ }^{84}$ The spectral range with which experimental data are recorded automatically limits the comparison to a wavelength region of practical interest. In contrast, when computed spectra are compared, a few lowfrequency normal modes with high ROA intensities tend to dominate spectral similarity. Not only is the pertinent spectral region not of practical interest but the use of the harmonic approximation is doubtful for this low-frequency region. For the purpose of the present work, we therefore found a quality measure based on reduced invariants, ${ }^{57}$ eq. 36 , which are devoid of frequency factors giving low-energy modes an inordinately large weight, more useful than measures derived from intensities. We have defined it as

$(J)=100 \sqrt{\frac{\sum_{p}^{N_{\text {vib }}}\left(J_{p}^{\mathrm{tr}}-J_{p}^{\mathrm{ref}}\right)^{2}}{\sum_{p}^{N_{\text {vib }}}\left(J_{p}^{\mathrm{ref}}\right)^{2}}}$.

$(J)$ represents a normalized percentage deviation of the reduced Raman and ROA invariants $J_{p}$ of a trial method (tr) with respect to a reference method (ref) summed over all $N_{\text {vib }}$ vibrations $p$ of a molecule. Average values were calculated seperately for the five chiral alcohols in Figure 1 and for their trimethylsilylethers.

Table 4 compares the deviations for different basis sets, as well as London and non-London orbitals for the alcohols. The invariant $J\left(a G^{\prime}\right)$ is clearly the most difficult to compute. It is interesting to note that this term is much more sensitive to the use of London orbitals than $J\left(\beta_{G}^{2}\right)$. In addition to the deviations of individual invariants, those of their linear

TABLE 4. Normalized mean square deviations (in per cent) relative to the results obtained with the analytical gradients and nonLondon orbitals using the aug-cc-pVDZ basis set for the secondary alcohols depicted in Figure 1, of the reduced invariants Jp, and of their combinations as they occur in the Raman back-, and in ROA back-, foreward-, and integral (int) scattering cross sections. $\mathrm{L}=$ London, $\mathrm{NL}=$ non-London orbitals. The numbers represent values averaged over those of the individual molecules

\begin{tabular}{lccrrrr}
\hline Label & Raman & ROA 180 & ROA 0 & ROA int & $J\left(a G^{\prime}\right)$ & $J\left(\beta_{G}^{2}\right)$ \\
\hline L rDPS & 2.8 & 8.1 & 13.4 & 8.9 & 15.0 & 8.6 \\
NL rDPS & 2.8 & 12.9 & 31.0 & 18.1 & 32.1 & 15.5 \\
L DPS & 3.2 & 4.2 & 4.7 & 4.4 & 5.4 & 4.5 \\
NL DPS & 3.2 & 5.7 & 14.4 & 7.1 & 16.2 & 7.7 \\
L aug-cc-pVDZ & 0.0 & 1.3 & 3.2 & 1.7 & 3.6 & 4.2 \\
\hline
\end{tabular}

TABLE 5. Normalized mean square deviations (in per cent) for the trimethylsilylethers of the secondary alcohols depicted in Fig. 1, of the reduced invariants Jp, and of their combinations as they occur in the Raman back-, and in ROA back-, foreward-, and integral (int) scattering cross sections. $\mathrm{L}=$ London, $\mathrm{NL}=$ non-London orbitals. The numbers represent values averaged over those of the individual molecules

\begin{tabular}{lccccrrr}
\hline Label & Raman & ROA 180 & ROA 0 & ROA int & $J\left(a G^{\prime}\right)$ & $J\left(\beta_{G}^{2}\right)$ & $J\left(\beta_{A}^{2}\right)$ \\
\hline L rDPS & 1.9 & 3.4 & 9.7 & 3.8 & 12.1 & 3.8 & 3.1 \\
Synth L rDPS & 9.5 & 28.5 & 69.2 & 30.2 & 73.7 & 29.7 & 34.2 \\
Synth NL rDPS & 9.5 & 29.0 & 76.7 & 31.5 & 79.6 & 30.6 \\
Synth L aug-cc-pVDZ & 8.8 & 28.9 & 67.2 & 30.9 & 71.0 & 30.4 \\
Synth NL aug-cc-pVDZ & 8.8 & 28.8 & 66.9 & 30.8 & 70.3 & 30.4 \\
\hline
\end{tabular}


combinations occurring in Raman and ROA intensity expressions are also included for selected scattering geometries. Deviations are largest, by far, for forward scattering where the salient ROA effects tend to be dominated by $a G^{\prime 85,86}$

The results of Table 4 make it obvious that rDPS, the smallest of the basis sets, profits most from using London orbitals. They are less important for DPS and even less so for aug-cc-pVDZ. However, we note that care must be exercised with $J\left(a G^{\prime}\right)$, and consequently, the benefit of using London orbitals is more pronounced for computed ROA forward than backward scattering spectra.

Table 5 lists results for the trimethylsilylethers of the five alcohols. The numbers permit a comparison of the expected quality of the results when either the fast rDPS basis set is used or when the electronic tensors are synthesized from a molecule's fragments. Clearly, synthesizing the molecule's electronic tensors degrades the quality of computed data more than the use of the small rDPS basis set. This is so even though the fragments were chosen as well-defined separate entities for which one does not expect excessive electronic interaction, namely, the chiral secondary alcohol part of the molecules and the achiral trimethylsilanol moiety.

As observed already for the alcohols, the changes are largest for $J\left(a G^{\prime}\right)$. This is true for the small rDPS basis set as well as when the tensors are synthesized from fragments. On the other hand, London orbitals make little difference when tensors are synthesized, even in the case of the small rDPS basis set. This is not unexpected as calculating the tensors from fragments indirectly distributes the gauge origin over the molecule.

The gain in computational efficiency achieved by synthesizing electronic tensors, though substantial, might at first sight not justify the degradation of the quality of computed results.

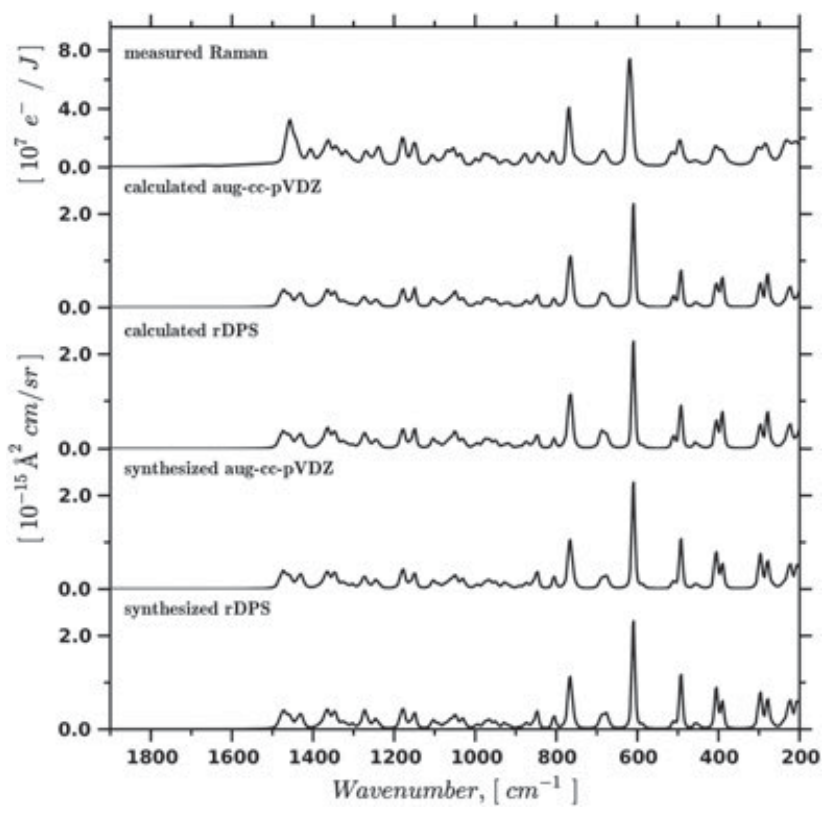

Fig. 2. Experimental and computed Raman backscattering spectra of the trimethylsilylether of menthol. From top to bottom: experimental spectrum, electronic tensor part of spectra computed with parameters as indicated with structure and force field calculated with r-pc-2. A linear correction has been applied to the calculated frequencies so that a shift of $0 \mathrm{~cm}^{-1}$ results for $300 \mathrm{~cm}^{-1}$ and of $-32 \mathrm{~cm}^{-1}$ for $1600 \mathrm{~cm}^{-1}$. The experimental data were recorded as follows: exposure time $60 \mathrm{~min}$, laser power at sample $400 \mathrm{~mW}$, exciting wavelength $532 \mathrm{~nm}$, instrumental resolution $7 \mathrm{~cm}^{-1}$. The curve has been slightly smoothed with the second-order symmetric five-point Savitzky-Golay filter.

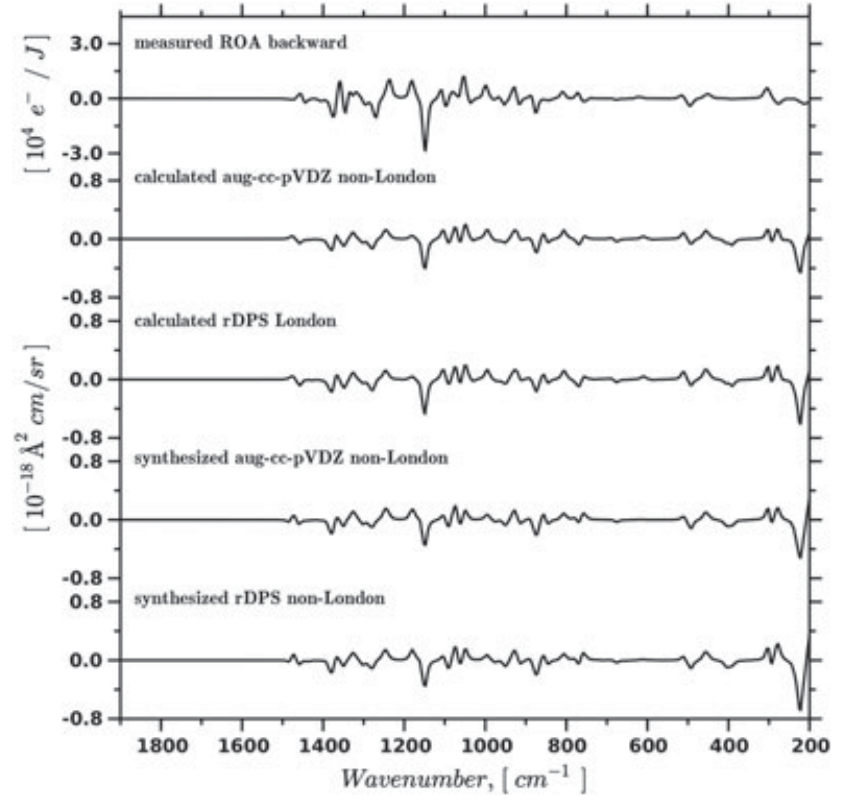

Fig. 3. Experimental and computed Raman optical activity (ROA) backscattering spectra of the trimethylsilylether of menthol. From top to bottom: experimental spectrum, computed spectra with electronic tensors calculated as indicated. See Figure 2 for details.

Speedups far larger than implied by the wall-clock times of Table 2 can, however, be achieved for larger molecules. Likewise, re-utilizing fragments either for different conformers of the same molecule, or for other molecules, leads to further computational savings. One has, moreover, to distinguish between the use of ROA for analytical and for spectroscopic purposes. For merely determining the absolute configuration of a molecule, in most cases, a high quality of the computed data is not required. For the detailed interpretation of the numereous bands in a vibrational ROA spectrum, it is desirable.

Figures 2 and 3 illustrate these aspects by comparing the results of different calculations of the backscattering spectrum of the trimethylsilylether of menthol with the measured one. From any of the computed ROA spectra, be it directly calculated with the aug-cc-pVDZ basis set, the minimalistic rDPS set, or synthesized from fragments with either of the two sets, the absolute configuration could, were it not known, easily be assigned beyond any doubt. Similarly, while conformational equilibria complicate the calculation of the ROA spectra for some of the secondary alcohols listed in Figure 1, and also for some of their trimethylsilylethers, it can safely be stated that when the absolute configuration can be assigned by a direct calculation of the whole molecule with aug-cc-pVDZ, then this is also possible by a direct calculation with the smaller rDPS set or by synthesizing the electronic tensor part for the spectra from fragments either with the aug-cc-pVDZ or the rDPS set.

\section{CONCLUSIONS}

A major obstacle to ROA's use for determining absolute configurations by the comparison of measured and computed spectra has in the past been its inefficient calculation. The bottleneck was the need to determine the gradients of electronic tensors by numerical differentiation. The analytical gradient scheme we propose provides an order of magnitude increase in computational speed for the typical organic molecules we use as examples. The formalism is based on a 
quasi-energy gradient and formulated in an atomic orbital basis. The approach is therefore well suited for taking advantage of the continuing improvements in linear scaling response theory that is currently taking place in the development of modern $a b$ initio methods. The implementation requires the solution of only 45 response equations, independently of the size of the molecule considered.

The use of efficient basis sets for the electronic tensor part provides another factor of two to three increase in speed if the DPS set developed for this purpose is employed, and 10 or more in the case of rDPS, as compared with the aug-cc-pVDZ we use as reference. These numbers refer to calculations at the TDHF level of theory and require a separate calculation of a molecule's structure and force field.

The present implementation of our formalism for analytical gradients is limited to non-London basis functions. Gaugeorigin dependence of computed results presents a problem for molecules substantially larger than those considered here when a small basis set such as rDPS is used, although these problems can be partially rectified using gauge transformation. ${ }^{19}$ Composing the tensors of larger molecules from those of fragments computed in smaller molecules solves this by distributing the gauge origin over these fragments. This moreover provides another substantial increase in computational speed, of the order of a factor of four or more already for the modestly sized molecules that we use as examples. Gains in speed by synthesizing electronic tensors are bound to increase with the size of the molecules.

The quality of electronic tensors is diminished by synthesizing them from fragments. Our quantitative error measure, based on the root-mean square deviation of reduced ROA invariants, suggests that the degradation can be substantial. In practical terms, though, as we show for ROA backscattering spectra, the qualitative appearance of the spectra undergoes little change, and the synthesized spectra easily permit the unambiguous assignment of a molecule's absolute configuration. Our results thus support recent studies into the accuracy of the tensor transfer techniques $^{87,88}$ that have been published in response to the recent concerns about the reliability of this appoach. ${ }^{89}$

The atomic orbital-based scheme for calculating the gradients of electronic tensors represents a major improvement over the numerical scheme. In combination with highly efficient basis sets, and the synthesis of these tensors for large molecules from separately calculated fragments, high computational speeds can now be reached for the electronic part of ROA calculations. Similar advances by mode tracking of vibrations of large molecules, ${ }^{90}$ and by synthesizing force fields from fragments, ${ }^{25}$ have recently been made for the computation of the vibrational part. Indeed, we believe the bottleneck in future ROA studies not to be the calculation of ROA invariants but rather the need to explore the conformational space of larger, flexible molecules.

\section{ACKNOWLEDGMENTS}

The trimethylsilylethers used as computational examples were synthesized by M. Lovchik, and their conformational anlysis, which will be reported elsewhere, was performed by E. Hasanova.

\section{LITERATURE CITED}

1. Barron LD. Molecular light scattering and optical activity. Cambridge: Cambridge University Press; 1982.

2. Barron LD, Buckingham AD. Rayleigh and Raman scattering from optically active molecules. Mol Phys 1971;20:1111-1119.
3. Barron LD, Bogaard MP, Buckingham AD. Raman Scattering of circularly polarized light by optically active molecules. J Am Chem Soc 1973;95:603-605.

4. Hug W, Kint S, Bailey GF, Scherer JR. Raman circular intensity differential spectroscopy. Spectra of $(-)-\alpha$-pinene and $(+)-\alpha$-phenylethylamine. J Am Chem Soc 1975;97:5589-5590.

5. Hug W, Haesler J. Is the vibrational optical activity of $(R)-\left[{ }^{2} \mathrm{H}_{1},{ }^{2} \mathrm{H}_{2},{ }^{2} \mathrm{H}_{3}\right]-$ neopentane measurable? Int J Quant Chem. 2005;104:695-715.

6. Haesler J, Schindelholz I, Riguet E, Bochet CG, Hug W. Absolute configuration of chirally deuterated neopentane. Nature 2007;446:526-529.

7. Bell AF, Hecht L, Barron LD. Vibrational Raman optical activity of DNA and RNA. J Am Chem Soc 1998;120:5820-5821.

8. Barron LD, Hecht L, Bell AF. Recent developments in Raman optical activity of biopolymers. Appl Spectrosc 1996;50:619-629.

9. Polavarapu PL, Deng Z. Structural determinations using vibrational Raman optical activity: from a single peptide group to $\beta$-turns. Faraday Discuss 1994;99:151-163.

10. Holzwarth G, Hsu EC, Mosher HS, Faulkner TR, Moscowitz A. Infrared circular dichroism of carbon-hydrogen and carbon-deuterium stretching modes. Observations. J Am Chem Soc. 1974;96:251-252.

11. Nafie LA, Cheng JC, Stephens PJ. Vibrational circular dichroism of 2,2,2trifluoro-1-phenylethanol. J Am Chem Soc 1975;97:3842-3843.

12. Stephens PJ. Theory of vibrational circular dichroism. J Phys Chem 1985;89:748-752.

13. Polavarapu PL. The absolute configuration of bromochlorofluoromethane. Angew Chemie, Int Ed. 2002;114:4726-4728.

14. Costante J, Hecht L, Polavarapu PL, Collet A, Barron LD. Absolute configuration of bromochlorofluoromethane from experimental and ab initio theoretical vibrational Raman optical activity. Angew Chemie, Int Ed. 1997;36:885-887.

15. Coriani S, Thorvaldsen AJ, Kristensen K, Jørgensen P. Variational response-function formulation of vibrational circular dichroism. Phys Chem Chem Phys 2011;13:4224-4229.

16. Stephens PJ. Theory of vibrational circular-dichroism. J Phys Chem 1985;89:748-752.

17. Bak KL, Jørgensen P, Helgaker T, Ruud K, Jensen HJA. Gauge-origin independent multiconfigurational self-consistent-field theory for vibrational circular-dichroism. J Chem Phys 1993;98:8873-8887.

18. Polavarapu PL. Ab initio vibrational Raman and Raman optical activity spectra. J Phys Chem 1990;94:8106-8112.

19. Bour P. Computations of the Raman optical activity via the sum-over-states expansions. J Comput Chem 2001;22:426-435.

20. Helgaker T, Ruud K, Bak KL, Jørgensen P, Olsen J. Vibrational Raman optical activity calculations using London atomic orbitals. Faraday Discuss 1994;99:165-180.

21. Ruud K, Helgaker T, Bour P. Gauge-origin independent density-functional theory calculations of vibrational Raman optical activity. J Phys Chem A 2002;106:7448-7455.

22. Herrmann C, Ruud K, Reiher M. Can Raman optical activity separate axial from local chirality? A theoretical study of helical deca-alanine. Chem Phys Chem. 2006;7:2189-2196.

23. Luber S, Reiher M. Intensity-carrying modes in Raman and Raman optical activity spectroscopy. Chem Phys Chem. 2009;10:2049-2057.

24. Jacob CR, Luber S, Reiher M. Analysis of secondary structure effects on the IR and Raman spectra of polypeptides in terms of localized vibrations. J Phys Chem B 2009;113:6558-6573.

25. Bouř $\mathrm{P}$, Sopková J, Bednárová L, Malon̆ $\mathrm{P}$, Keiderling TA. Transfer of molecular property tensors in cartesian coordinates: a new algorithm for simulation of vibrational spectra. J Comput Chem 1997;18:646-659.

26. Kapitán J, Baumruk V, Bour P. Demonstration of the ring conformation in polyproline by the Raman optical activity. J Am Chem Soc 2006;128: 2438-2443.

27. Luber S, Reiher M. Theoretical Raman optical activity study of the $\beta$-domain of rat metallothionein. J Phys Chem B 2010;114:1057-1063.

28. Liégeois V, Ruud K, Champagne B. An analytical derivative procedure for the calculation of vibrational Raman optical activity spectra. J Chem Phys 2007;127:204105.

29. Thorvaldsen AJ, Ruud K, Kristensen K, Jørgensen P, Coriani S. A density matrix-based quasienergy formulation of the Kohn-Sham density functional response theory using perturbation- and time-dependent basis sets. J Chem Phys 2008;129:214108. 
30. Hudecova J, Kapitan J, Baumruk V, Hammer RP, Keiderling TA, Bour̆ P. Side chain and flexibility contributions to the Raman optical activity spectra of a model cyclic hexapeptide. J Phys Chem A 2010;114: $7642-7651$.

31. Yamamoto S, Straka M, Watarai H, Bouř P. Formation and structure of the potassium complex of valinomycin in solution studied by Raman optical activity spectroscopy. Phys Chem Chem Phys 2010;12:11021-11032.

32. Yamamoto S, Watarai H, Bouř P. Monitoring the backbone conformation of valinomycin by Raman optical activity. Chemphyschem 2011;12: 1509-1518.

33. Yamamoto S, Bour P. On the limited precision of transfer of molecular optical activity tensors. Collect Czech Chem Commun. 2011;76:567-583.

34. Sebestik J, Bouř P. Raman optical activity of methyloxirane gas and liquid. J Phys Chem Lett. 2011;2:498-502.

35. Profant V, Baumruk V, Li X, Safarik M, Bour P. Tracking of the polyproline folding by density functional computations and Raman optical activity spectra. J Phys Chem B 2011;115:15079-15089.

36. Hudecova J, Hopmann KH, Bour P. Correction of vibrational broadening in molecular dynamics clusters with the normal mode optimization method. J Phys Chem B 2012;116:336-342.

37. Hopmann KH, Sebestik J, Novotna J, Stensen W, Urbanova M, Svenson J, Svendsen JS, Boulv\{r\} P, Ruud K, Determining the absolute configuration of two marine compounds using vibrational chiroptical spectroscopy. J Org Chem 2012;77:858-869.

38. Johannessen C, Hecht L, Merten C. Comparative study of measured and computed Raman optical activity of a chiral transition metal complex. Chemphyschem 2011;12:1419-1421.

39. Merten C, Barron LD, Hecht L, Johannessen C. Determination of the helical screw sense and side-group chirality of a synthetic chiral polymer from Raman optical activity. Angew Chem. 2011;50:9973-9976.

40. Cheeseman JR, Shaik MS, Popelier PLA, Blanch EW. Calculation of Raman optical activity spectra of methyl- $\beta$-D-glucose incorporating a full molecular dynamics simulation of hydration effects. J Am Chem Soc 2011;133:4991-4997.

41. Hopmann KH, Ruud K, Pecul M, Kudelski A, Dracinsky M, Bouř P. Explicit versus implicit solvent modeling of Raman optical activity spectra. J Phys Chem B 2011;115:4128-4137.

42. Chruszcz-Lipska K, Blanch EW. In situ analysis of chiral components of pichtae essential oil by means of ROA spectroscopy: experimental and theoretical Raman and ROA spectra of bornyl acetate. J Raman Spectrosc. 2012;43:286-293.

43. Coriani S, Høst S, Jansik B, Thøgersen L, Olsen J, Jørgensen P, Reine S, Pawlowski F, Helgaker T, Sa $\{$ \} $\}$ ek P, Linear-scaling implementation of molecular response theory in self-consistent field electronic-structure theory. J Chem Phys 2007;126:154108.

44. Kussmann J, Ochsenfeld C. A density matrix-based method for the linearscaling calculation of dynamic second- and third-order properties at the Hartree-Fock and Kohn-Sham density functional theory levels. J Chem Phys 2007;127:204103.

45. Niklasson AM, Weber V. Linear scaling density matrix perturbation theory for basis-set-dependent quantum response calculations: an orthogonal formulation. J Chem Phys 2007;127:064105.

46. Niklasson AM, Challacombe M. Density matrix perturbation theory. Phys Rev Lett 2004;92:193001.

47. Weber V, Niklasson AM, Challacombe M. Ab Initio linear scaling response theory: electric polarizability by perturbed projection. Phys Rev Lett 2004;92:193002.

48. Izmaylov AF, Brothers EN, Scuseria GE. Linear-scaling calculation of static and dynamic polarizabilities in Hartree-Fock and density functional theory for periodic systems. J Chem Phys 2006;125:224105.

49. Bast R, Ekström U, Gao B, Helgaker T, Ruud K, Thorvaldsen AJ. The ab initio calculation of molecular electric, magnetic and geometric properties. Phys Chem Chem Phys 2011;13:2627-2651.

50. Helgaker T, Coriani S, Jørgensen P, Kristensen K, Olsen J, Ruud K. Recent advances in wave function-based methods of molecular-property calculations. Chem Rev 2012;112:543-631.

51. Thorvaldsen AJ, Ferrighi L, Ruud K, Ågren H, Jørgensen P, Coriani S. Analytic ab initio calculations of coherent anti-Stokes Raman scattering (CARS). Phys Chem Chem Phys 2009;11:2293-2304.

52. Thorvaldsen AJ, Ruud K, JJaszul'\{n\}ski M. Analytic calculations of vibrational hyperpolarizabilities in the atomic orbital basis. J Chem Phys A. 2008;112:11942-11950.
53. Sałek P, Høst S, Thøgersen L, Jørgensen P, Manninen P, Olsen J, Manninen P, Olsen J, Jansł' $\backslash$ i\}k B, Reine S, Paw\{V\}owski F, Tellgren E, Helgaker T, Coriani S. Linear-scaling implementation of molecular electronic self-consistent field theory. J Chem Phys 2007;126:114110.

54. Helgaker T, Jørgensen P. Configuration-interaction energy derivatives in a fully variational formulation. Theor Chim Acta. 1989;75:111-127.

55. Kristensen K, Jørgensen P, Thorvaldsen AJ, Helgaker T. Efficient elimination of response parameters in molecular property calculations for variational and nonvariational energies. J Chem Phys 2008;129:214103.

56. Hug W. Visualizing Raman and Raman optical activity generation in polyatomic molecules. Chem Phys. 2001;264:53-69.

57. Hug W. In: Continuum solvation models in chemical physics. Chichester: John Wiley and Sons Ltd.; 2007. p. 220.

58. Fedorovsky M. A program for anayzing vibrational motion and vibrational spectra; 2007. http://pyvib2.sourceforge.net.

59. Fedorovsky M. Raman optical activity: from analyzing to synthesizing vibrational spectra. Switzerland: University of Fribourg; 2008.

60. Buckingham $\mathrm{AD}$, Longuet-Higgins $\mathrm{HC}$. The quadrupole moments of dipolar molecules. Mol Phys. 1968;14:63-72.

61. Barron LD, Buckingham AD. Simple two-group model for Rayleigh and Raman optical activity. J Am Chem Soc 1974;96:4769-4773.

62. Hug W. In: Handbook of vibrational spectroscopy. vol. 1. Chichester: John Wiley and Sons Ltd.; 2002. p. 745.

63. Pulay P. Ab initio calculation of force constants and equilibrium geometries in polyatomic molecules. Mol Phys. 1969;17:197-204.

64. Reine S, Tellgren E, Krapp A, Kjærgaard T, Helgaker T, Jansik B, Jans〉' 〈i\} $\mathrm{k}$ B, Høst S, Sa $\{$ \} \}ek P. Variational and robust density fitting of four-center two-electron integrals in local metrics. J Chem Phys 2008;129:104101.

65. Hug W, Fedorovsky M. Characterizing vibrational motion beyond internal coordinates. Theor Chem Acc. 2008;119:113-131.

66. Kneller JR. Superposition of molecular structures using quaternions. Mol Simul. 1991;7:113-119.

67. Sverdlov LM, Kovner MA, Krainov EP. Vibrational spectra of polyatomic molecules. John Wiley and Sons, New York; 1974.

68. Hug W, Zuber G, de Meijere A, Khlebnikov AF, Hansen HJ. Raman Optical Activity of a Purely $\sigma$-Bonded Helical Chromophore: (-)-(M)- $\sigma-[4]$ Helicene. Helv Chim Acta 2001;84:1-21.

69. DALTON, A molecular electronic structure program, Release 2.0; 2005 http://www.kjemi.uio.no/software/dalton/dalton.html.

70. Hamprecht FA, Cohen AJ, Tozer DJ, Handy NC. Development and assessment of new exchange-correlation functionals. J Chem Phys 1998;109:6264-6271.

71. Jensen F. Polarization consistent basis sets: principles. J Chem Phys 2001;115:9113-9125.

72. Jensen F. Polarization consistent basis sets. II. Estimating the Kohn-Sham basis set limit. J Chem Phys 2002;116:7372-7379.

73. Frisch MJ, Trucks GW, Schlegel HB, Scuseria GE, Robb MA, Cheeseman JR, Montgomery JrJA, Vreven T, Kudin KN, Burant JC, Millam JM, Iyengar SS, Tomasi J, Barone V, Mennucci B, Cossi M, Scalmani G, Rega N, Petersson GA, Nakatsuji H, Hada M, Ehara M, Toyota K, Fukuda R, Hasegawa J, Ishida M, Nakajima T, Honda Y, Kitao O, Nakai H, Klene M, Li X, Knox JE, Hratchian HP, Cross JB, Bakken V, Adamo C, Jaramillo J, Gomperts R, Stratmann RE, Yazyev O, Austin AJ, Cammi R, Pomelli C, Ochterski JW, Ayala PY, Morokuma K, Voth GA, Salvador P, Dannenberg JJ, Zakrzewski VG, Dapprich S, Daniels AD, Strain MC, Farkas O, Malick DK Rabuck AD, Raghavachari K, Foresman JB, Ortiz JV, Cui Q, Baboul AG, Clifford S, Cioslowski J, Stefanov BB, Liu G, Liashenko A, Piskorz P, Komaromi I, Martin RL, Fox DJ, Keith T, Al-Laham MA, Peng CY, Nanayakkara A, Challacombe M, Gill PMW, Johnson B, Chen W, Wong MW, Gonzalez C, Pople JA. Gaussian 03, Revision D.02;. Gaussian, Inc., Wallingford, CT, 2004.

74. Woon DE, Dunning TH. Gaussian basis sets for use in correlated molecular calculations. IV. Calculation of static electrical response properties. J Chem Phys 1994;100:2975-2988.

75. Dunning TH. Gaussian basis sets for use in correlated molecular calculations. I. The atoms boron through neon and hydrogen. J Chem Phys 1989;90:1007-1023.

76. Zuber G. Méthodes de visualisation et calcul de l'activité optique Raman vibrationnelle. University of Fribourg, Switzerland; 2004.

77. Binkley JS, Pople JA, Hehre WJ. Self-consistent molecular orbital methods. 21. Small split-valence basis sets for first-row elements. J Am Chem Soc 1980;102:939-947. 
78. Zuber G, Hug W. Rarefied basis sets for the calculation of optical tensors. 1. The importance of gradients on hydrogen atoms for the Raman scattering tensor. J Phys Chem A 2004;108:2108-2118.

79. Norman P, Jonsson D, Ågren H, Dahle P, Ruud K, Helgaker T, Koch H Efficient parallel implementation of response theory: calculations of the second hyperpolarizability of polyacenes. Chem Phys Lett. 1996;253:1-7.

80. Fossgård E, Ruud K. Superlinear scaling in master-slave quantum chemical calculations using in-core storage of two-electron integrals. J Comput Chem 2006;27:326-333.

81. Reiher M, Liegeois V, Ruud K. Basis set and density functional dependence of vibrational Raman optical activity calculations. J Phys Chem A 2005;109:7567-7574.

82. Zuber G, Goldsmith MR, Beratan DN, Wipf P. Towards Raman optical activity calculations of large molecules. Chem Phys Chem. 2005;6: 595-597.

83. Luber S, Herrmann C, Reiher M. Relevance of the electric-dipole-electricquadrupole contribution to Raman optical activity spectra. J Phys Chem B 2008;112:2218-2232.
84. Fedorovsky M, Gerlach H, Hug W. Absolute conformations of the (-)-[9](2,5)pyridinophane molecule. Helv Chim Acta 2009;92: 1451-1465.

85. Barron LD, Hecht L, Gargaro AR, Hug W. Vibrational Raman optical activity in forward scattering: trans-pinane and $\beta$-pinene. J Raman Spectrosc. 1990;21:375-379.

86. Hug W, Haesler J, Kozhushkov SI, de Meijere A. 1,4-Dimethylenespiropentane: a unique model system for studying fermi resonance in Raman optical activity. Chem Phys Chem. 2007;8:1161-1169.

87. Yamamoto S, Kaminsky J, Bouř P. Anal Chem 2012;84:2440.

88. Yamamoto S, Li X, Ruud K, Bouř P. Transferability of various molecular property tensors in vibrational spectroscopy. J Chem Theory Comput. 2012;8:977-985.

89. Bieler NS, Haag MP, Jacob CR, Reiher M. Analysis of the cartesian tensor transfer method for calculating vibrational spectra of polypeptides. J Chem Theory Comput. 2011;7:1867-1881.

90. Reiher M, Neugebauer J. A mode-selective quantum chemical method for tracking molecular vibrations applied to functionalized carbon nanotubes. J Chem Phys 2003;118:1634-1641. 